\title{
Plants: a tool box of cell-based assays
}

\author{
Vivien Marx
}

\begin{abstract}
Cell-based assays are less routine for plant biologists than for researchers who work with animal or human cells, but that is changing.
\end{abstract}

The Trembling Giant is a forest of 50,000 slender aspens that gets its name from the sound of its trees' leaves rustling in the wind. These 80,000-year-old trees in Utah's Fishlake National Forest are a single plant clone. All of the trees are genetically identical because they have grown asexually, through a process called suckering, from one parental root. Plant biologist David Galbraith of the University of Arizona wonders how a clonal plant with identical somatic cells could have survived predation and global climate change for so long, and which cellular mechanisms mattered in its survival. There's no answer to this question yet, he says. Another question that intrigues him is whether individual somatic cells of plants are identical or differ genetically, epigenetically or in some other way.

Such questions call for cell-based assays, which might not seem to be as prominent in plant-based labs as in labs focused on animal or human cells. But Philip Benfey, a plant biologist at Duke University who began his career working with animal cells, says, "I don't think that there's a sense that we just can't do certain

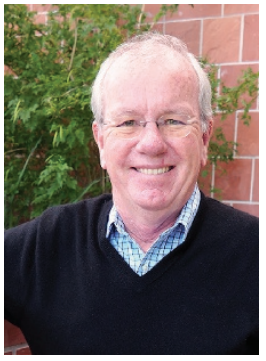
things that are being done in the animal field, because we don't have a technique." Plant biologists have assay options with which to study cells in their normal or near-normal context.

As is true for Labs should apply stringent quality control measures when generating protoplasts, says David Galbraith. and primary cells. Mammalian cell line biorepositories were created with massive effort and funding, notably from the US National Institutes of Health, says Benfey. Even without these resources, plant biologists have long applied genetic techniques and cellular analysis to explore many basic and applied research questions. They can carry out high-throughput molecular assays using protoplasts, though these engender some controversy, and they can obtain tissue- or cell type-specific transcriptional or epigenetic profiles by sorting labeled protoplasts or nuclei and profiling chromatin or RNA.

\section{Protoplasts: tools without walls}

Despite many technical advances, labs have learned only a fraction of plant biology through conventional mutant characterization and transgenic plant analyses, says Jen Sheen, a plant biologist at Massachusetts General Hospital. The increasing number of plant genome sequences available has, she says, ushered in a need for high-throughput methods to study gene function and regulation and explore genotype-phenotype relationships, especially for mutants that are lethal or have complicated phenotypes. Making protoplasts, or 'protoplasting', is her high-throughput method of choice to explore such questions.

Protoplasts are plant cells from which the cell walls have been enzymatically removed. Protoplasts do not divide. Some scientists say protoplasts skew the view of the in planta condition, but others say protoplasts are ideal for molecular genetic studies because they retain their cell type traits after being isolated from a plant. Protoplast-based assays are transient; depending on the lab and experiment, the protoplasts survive half a day or several days. As Galbraith explains, now that labs can increasingly manipulate protoplasts

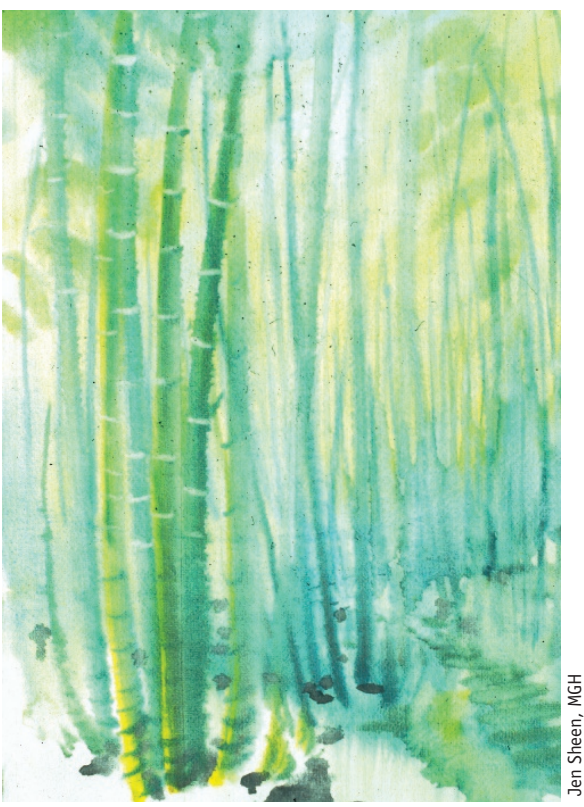

Plant biologists can perform assays similar to those used on animal and human cells.

with molecular tools such as transfection and gene editing, a more detailed and sophisticated understanding of protoplasts will emerge, as will ways to manipulate them to achieve desired goals. Scientists recently developed a DNA-free system for editing plant genomes that uses protoplasts and CRISPR-Cas9. The scientists made targeted modifications in six genes from four plant species ${ }^{1}$. More such experiments await.

Given that protoplasts can be isolated from any number of plant tissues and that they maintain their cellular identity, they can be used to explore all sorts of questions specific to cell type, say plant biologists Francesca Quattrocchio and Ronald Koes at the University of Amsterdam in a joint note. This husband-and-wife research team say many scientists think protoplasts inadequately 


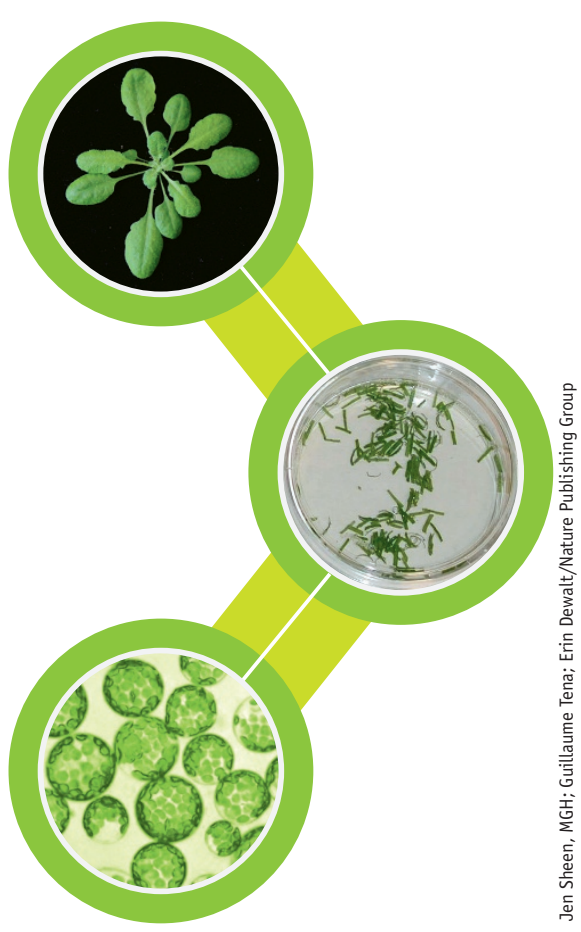

To obtain protoplasts, which are plant cells without cell walls, plant tissue is treated with enzymes. For leaf protoplasts, researchers first manually cut a leaf into strips.

reflect in planta conditions. They counter that in vitro protoplasts are more similar to the cells in intact plants than animal cell cultures are to the organisms from which they were taken or derived.

Protoplasts are useful in tracking intracellular processes because they are easy to transform and to image with confocal microscopy, say Quattrocchio and Koes. In their view, protoplasts are well suited for studying factors that influence gene expression, the appearance of membrane structures and the accumulation of compounds, as well as protein-protein interactions, protein localization and gene function.

Researchers are tempted to use the easiest tissue source of protoplasts and assume that the choice is meaningless, say Quattrocchio and Koes. Yet the choice matters: some proteins do not localize to the same compartment in all cells, some promoters are not active in all cell types, and some protein-protein interactions do not occur in all cells. The researchers therefore recommend harvesting protoplasts from cells in which the gene, protein or membrane of interest is normally present $^{2}$.

Labs can use protoplasts to study gene expression or membrane characteristics, but it is important to remember that protoplast isolation often leads to a mix of cell types, say Quattrocchio and Koes. Researchers can study transcription factors using cells in which a promoter is inactive and then try to activate it. Quattrocchio and Koes say that they have kept protoplasts alive for five days in their lab. As a transient assay, protoplasting avoids callus development, in which protoplasts de-differentiate and lose their cell type specificity.

The versatility of protoplasts, says Sheen, helps scientists explore physiologically relevant mechanisms, such as those involved in gene regulation and signal transduction, in plant 'primary' cells. Such work in intact plants takes months to years; in protoplasts it takes hours to days. Sheen has been working with protoplasts since 1987, when mutant cloning and transgenic plant technology were in their infancy ${ }^{3}$. She was drawn to protoplasts, to the chagrin of her advisers and prominent plant biologists she knew who feared the assay was too "artificial," she recalls. She remains a protoplast proponent for many kinds of experiments.

\section{Are they stressed?}

Protoplasts are cumbersome to isolate in quantity, says Detlef Weigel, a researcher at the Max Planck Institute for Developmental Biology in Tübingen, Germany. He studies the genetic barriers to mating in plant flowering mechanisms and the ways plants adapt to changing environments, and leads, along with two colleagues, the 1001 Genomes Project, which is cataloging genetic variation in Arabidopsis. In many ways, he says, protoplasts are the equivalent of primary cell culture of animal and human cells; but, because they do not divide and cannot be amplified, protoplasts are "of limited usefulness."

The literature on protoplast assays dates back to the early days of clonally propagating plants in cell culture, says Michael Blatt, a plant biologist at the University of Glasgow and editor-in-chief of the journal Plant Physiology. If one includes mechanical methods of producing protoplasts from the giant alga Chara, protoplasting dates back even further, to the 1920s and 1930s.

To study membrane trafficking in animal cells with, for example, patch-clamp experiments, says Blatt, researchers strip the extracellular matrix away from the membrane. The cell maintains an osmotic balance between inside and outside. Each plant cell is surrounded by a cellulose wall, within which turgor and high salt content are maintained. "If you remove the cell wall, they go 'pop,"
Blatt says. Once removed from plant tissue, protoplasts must be osmotically stabilized. "You could ask the question," he says, "is this actually representative of what actually goes on in the cell when it's inside a cellulosic matrix?" When a protoplast is stressed, he says, it secretes a pectin matrix to rebuild the cell wall and restabilize itself.

Despite this stress, Blatt says, protoplasts can be used in many types of experiments, including those characterizing factors involved in membrane trafficking and secretion. But researchers should remember that this is not the in planta condition, he says, because "the cell is doing its damndest to reform its cell wall as quickly as it can."

In looking for signs of stress in their protoplasts, Quattrocchio and Koes say, they have found no differences between protoplasts and plant cells that have been transformed by agroinfiltration, a transient assay in which the bacterium Agrobacterium tumefaciens is used to deliver genes into the plant genome. The researchers have studied various characteristics of petal and leaf cells, such as the activity of specific promoters and genes, the presence of membrane compartments and membrane localization and have looked at soluble proteins in different cell compartments. Stress is certainly a possibility, they say, but it is not dramatic and does not appear to affect any of the processes they study.

"I was always concerned that the process of protoplast production resulted in patterns of gene expression and signaling that might reflect the stresses of this process," says Galbraith. He has found that some gene regulation networks are affected by protoplasting. Benfey says that although protoplasting shocks the cells and leads to some change, a "remarkable amount of their RNA state" remains constant. Around 1-5\% of RNA changes in the 1.5-2 hours between starting enzymatic digestion and running
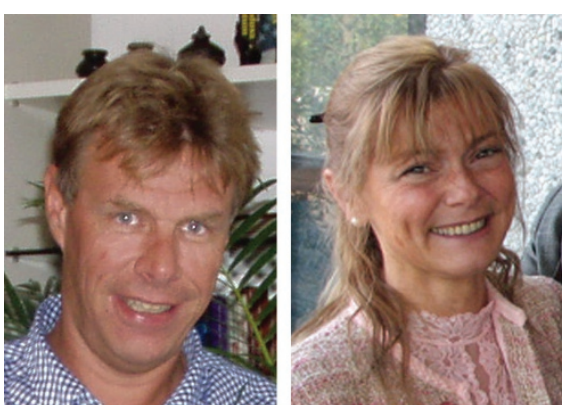

The easiest source of protoplasts might not be the best, say Francesca Quattrocchio and Ron Koes. 


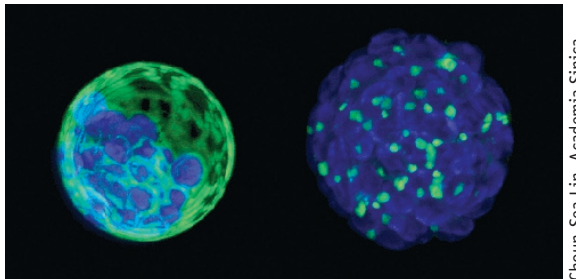

Protoplasts labeled with fluorescent proteins show the vacuoles in blue (left) and peroxisomes (right) in green.

the protoplasts through a cell sorter, Benfey says. Some of that change might be due to the cell-sorting process itself.

Protoplasts start to make cell walls after around three days, says Benfey. To study protoplast changes, he has looked at expression levels in a light-harvesting protein in leaf protoplasts. "You could just see it fading over about 6 hours; after 12 hours it was essentially gone," he says. At that point and in that one aspect, the cell had changed its identity. Given these findings, he says, once cells exit the sorter they go straight into extraction buffer, which essentially preserves them in their state at that time.

For labs studying primary metabolites or amino acids, which change rapidly, there is probably insufficient time for an adequate experiment, says Benfey. But time might suffice for studying secondary metabolites such as lipids and proteins. "Many proteins seem to stay the same; that is, they don't turn over, they don't get degraded in that same time frame," he says.

\section{Making protoplasting easier}

'Operator skill', 'high skill' and 'advanced skills' are terms that pepper the protoplasting protocols. To generate protoplasts, scientists need to manually cut a leaf into strips $0.5-1 \mathrm{~mm}$ wide without crushing the leaf or macerating the cells. That is challenging and may yield too few usable protoplasts. For years, scientists had to use plants grown in sterile suspension cultures, which excluded certain natural strains known as ecotypes. Over time, easier approaches have emerged, and researchers can now get protoplasts from potted plants grown in soil. Scientists at Academia Sinica in Taiwan and Purdue University have developed a method, called 'tape-Arabidopsis sandwich', in which they use two kinds of tape to remove the epidermal layer of a leaf ${ }^{4}$. No leaf cutting is needed; the protoplasts are released when the leaf is incubated in an enzyme solution.

Choun-Sea Lin, who runs the agricultural biotechnology research core at Academia Sinica and was the lead author of the study ${ }^{4}$, says he got the idea for the tape-Arabidopsis sandwich method while playing with his son as they unpacked taped boxes. The approach reduces the time it takes to generate protoplasts from five hours to one. Instead of one day, transfection takes two hours or fewer. And cutting leaves correctly has been a protoplasting challenge, says Sheen, making the tape-Arabidopsis sandwich a useful advance.

When protoplasting, it's critical for labs to grow and recognize healthy plants, says Sheen, and they need to take the time to test and find the optimal plant and organ growth stage for their starting material. Galbraith is concerned that labs generating protoplasts may not always apply stringent quality-control measures or know which traits to look for. And, he says, scientists too rarely use microscopy to make sure the protoplasts are healthy.

\section{Expanding the tool kit}

Protoplasts have to be maintained in medium that is high in sodium and generally stressful for them, says Lin. "That is why many scientists don't like to use protoplasts and prefer to use laser-capture microdissection for expression profiling study or use AGROBEST for transient transformation," he says. AGROBEST (Agrobacterium-mediated enhanced seedling transformation) is a transient expression assay that can be used with different plant tissues and for systematic screens.

Lin says that he and his team have used laser-capture microdissection in plant cells but that it is not robust for isolating sufficient RNA for expression studies. Until RNA isolation and sequencing technology improve, he says, protoplasting is most advantageous for studying gene expression. And when looking at subcellular localization after transient transformation, image quality with protoplasting is much better than with AGROBEST, he says. Lin and his group are setting up an in-house protoplasting service for transient transformation experiments, CRISPR assays and studying protein-protein interactions.

As protoplasting methods evolve, Lin hopes to use them to study the plant root and do more single-cell RNA sequencing. To generate protoplasts for transformation experiments conveniently, he would also like to be able to isolate protoplasts from all root types, not just young roots.

Working with protoplasts is not easy, which may explain some of the skepticism in the plant community. A key question, says Sabeeha Merchant, a plant biologist at UCLA and editor-in-chief of The Plant Cell, is whether the community is skeptical because they cannot get the assays to work or are concerned that the bulk of the conclusions from protoplast-based assays might turn out to be flawed. Merchant has not worked with protoplasts; her lab uses Chlamydomonas as a reference organism for studying certain aspects of metabolism in green plants, because microorganisms allow studies with a uniform population of cells.

Merchant and her team have used pulsechase methods to follow the uptake and turnover of labeled compounds in a cell. Pulse-chase methods are more straightforward with homogeneous populations of cells that grow and divide than with multicellular organisms. But microbial models cannot be used to study aspects of plant biology such

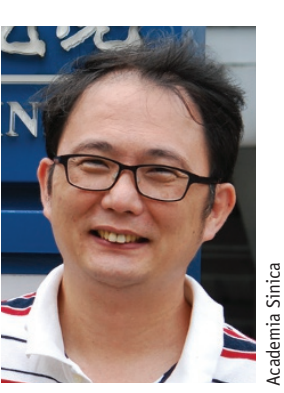

It is quicker and easier to generate protoplasts with the tape-Arabidopsis sandwich method, says Choun-Sea Lin. as development or hormone signaling, so there is a need for cell-based assays in a multicellular organism. "The use of protoplasts is one possible route," Merchant says. Other options include use of cell type-specific fluorescent markers, laser-capture microdissection to cut cells out of tissue, and isolation of nuclei tagged in specific cell types (INTACT), developed by Steve Henikoff and Roger Deal at Fred Hutchinson Cancer Research Center ${ }^{5}$.

\section{Molecular profiles from tissues}

Molecular profiling of cell types helps with many research questions in plant biology. Using flow cytometry to sort fluorescently labeled protoplasts from tissue for microarray hybridization or RNA sequencing, Benfey and his colleagues have created a cellular-resolution-level atlas of gene expression in the Arabidopsis root ${ }^{6}$. They have found that expression levels are comparable to those in the intact plant. What he likes about the root, says Benfey, is that it is translucent, has little autofluorescence and allows different cell types to be seen easily. Cell types are arranged concentrically, and each 
root contains cells at various developmental stages. Protoplast sorting based on cell-typespecific fluorescent labels, he says, is almost like peering into a beating heart and discerning myocardial from vascular cells by their expression patterns. The assay's resolution is now being improved as single-cell techniques such as transcriptional and epigenomic analysis continue to develop in a promising manner, he says.

Laser-capture microdissection, in which a laser is manually directed to dissect cells from tissue on a microscope slide, can also be used for gene-expression analysis, but it is timeconsuming, especially for single-cell analysis, says Galbraith. And the method works only for cells that have a distinct phenotype. "If you have a cell type that doesn't have a visible phenotype, then you can't dissect it," he says.

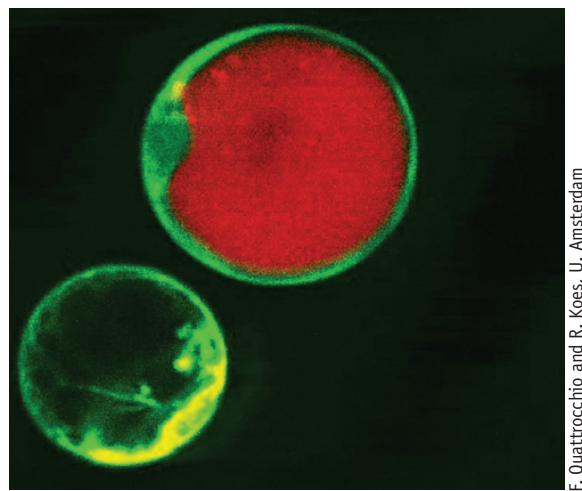

Discerning petunia protoplasts with confocal microscopy. One protoplast, from the epidermis, contains anthocyanin pigments (red), whereas the one from the mesophyll has no pigment (lower left). Both express GFP fused to a cytoplasmic protein (green).
Benfey used laser-capture microdissection extensively at GrassRoots Biotechnology, a company he founded, which was acquired by Monsanto in 2013. What is challenging about the method is that cells have to be fixed or cryofixed and sectioned, and a scientist then has to identify what to cut out of the tissue. "It's just an incredibly laborious process," he says. Given that RNA extraction and analysis can now be done at the single-cell level, researchers can use laser-capture microdissection for RNA sequencing. But for metabolomics or proteomic analysis, he says, "that's just an impossibility."

Another alternative to sorting fluorescent protoplasts is INTACT, a method that extracts nuclei from plant organs and enriches for biotin-labeled nuclei in specific cell types using affinity purification. Weigel's sense is that INTACT is "coming into its own.” INTACT works, says Galbraith, but only to examine populations of nuclei, not single nuclei. He adds that a bacterial biotinprotein ligase must be active and coexpressed with a nuclear-targeting fusion protein in every cell of interest for nuclear labeling to work.

To study individual cells, Galbraith and his team use flow sorting to isolate single nuclei, then they perform single-cell RNA sequencing. He prefers sorting nuclei from tissue homogenates rather than protoplasts because breaking the cells open arrests cell signaling pathways ${ }^{7}$. "We have also shown that the poly(A) RNA of nuclei is very similar to that of the whole cell," Galbraith says. $\mathrm{He}$ and his team have analyzed transcription across the entire genome using the poly(A)

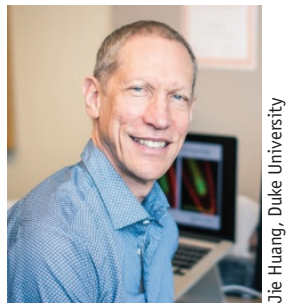

RNA contained in a single nucleus. Benfey, however, wonders whether the components of RNA found in the nucleus are indeed like the RNA in the cytoplasm. More

What he likes about the root, says Philip Benfey, is that it is translucent and has little autofluorescence, and different cell types are easy to spot. generally, his sense is that INTACT is most valuable for epigenomic studies and chromatin immunoprecipitation experiments. "Here it should be the method of choice," he says of INTACT.

Scientists will encounter differences of opinion about when to choose protoplast sorting, INTACT or laser-capture microdissection, says Benfey. In all cases, he says, the same best practices for studies with animal cells apply to in vitro assays on plant cells: "you really have to go back and test in the intact plant."

1. Woo, J.W. et al. Nat. Biotechnol. 33, 1162-1164 (2015).

2. Faraco, M. et al. Plant Physiol. 156, 474-478 (2011).

3. Sheen, J. Plant Physiol. 127, 1466-1475 (2001).

4. Wu, F.H. et al. Plant Methods 5, 16 (2009).

5. Deal, R.B. \& Henikoff, S. Nat. Protoc. 6, 56-68 (2011).

6. Brady, S.M. et al. Science 318, 801-806 (2007).

7. Galbraith, D.W. et al. Science 220,1049-1051 (1983).

Vivien Marx is technology editor for Nature Methods (v.marx@us.nature.com). 